\title{
Expanding the hospital incident command system with a physician-centric role during a pandemic: The role of the physician clinical support supervisor
}

\author{
Jason Persoff*1 ${ }^{*}$ Hemali Patel ${ }^{1}$, Sarguni Singh ${ }^{1}$, Cathy Ehrenfeucht ${ }^{2}$, Jean Kutner ${ }^{1}$, Charles Little ${ }^{1}$, Sarah E. Tevis ${ }^{1}$ \\ ${ }^{1}$ University of Colorado School of Medicine, Aurora, CO, United States \\ ${ }^{2}$ University of Colorado Hospital, Aurora, CO, United States
}

Received: May 11, 2020

DOI: $10.5430 /$ jha.v9n3p7
Accepted: May 27, 2020

Online Published: June 5, 2020

URL: https://doi.org/10.5430/jha.v9n3p7

\begin{abstract}
The SARS-CoV-2 (COVID-19) pandemic is the largest mass effect incident in a century, requiring hospitals to consider how best to adapt the Hospital Incident Command System for a sustained pandemic. Given the scope of the pandemic a central position is required to improve the flow of information to providers, the flow from providers to the Incident Commander, and the overall coordination between diverse service lines and specialties. We describe a novel position, the Physician Clinical Support Supervisor, a position that has three primary functions during disaster operations: liaison, coordinator, and advocate. This role proved critical in day-to-day operations and facilitated highly effective communication up and down the chain of command, created a single point-person to coordinate multiple service lines and specialties, and served as a primary advocate for front-line workers and command.
\end{abstract}

Key Words: Emergency preparedness, SARS-CoV-2, Hospital Incident Command System, Disaster response

\section{INTRODUCTION}

Mass effect incidents-sustained crises often due to loss of infrastructure or pandemic infection-have the potential to create an acute and sustained surge of patients. Mass effect incidents can overwhelm available hospital resources and personnel, deplete local and regional stockpiles, and overwhelm Federal supplies such as with the SARS-CoV-2 (COVID-19) pandemic. ${ }^{[1-4]}$ The pandemic is the largest mass effect incident in a century, and it has placed hospitalists directly in the front lines of coordinating care for patients often outside their normal scope of practice. As described in the work we have published, we believe hospitalists are essential in the hospital incident command system (HICS) as part of an all-hazards approach to all disasters. ${ }^{[5]}$ The COVID-19 pandemic has solidified this vital role called the Physician Clinical Support Supervisor (PCSS) at our institution within the HICS structure and serves three main roles: liaison, coordinator, and advocate.

\section{DESCRIPTION OF ROLE}

\subsection{Background}

The National Response Framework (NRF) was created as part of a national system to coordinate approaches to all disasters spanning both military and civilian jurisdictions through

\footnotetext{
*Correspondence: Jason Persoff; Email: jason.persoff@cuanschutz.edu; Address: University of Colorado, Mail Stop F782, 12401 E 17th Ave, Aurora CO 80045, United States.
} 
the National Incident Management System (NIMS). ${ }^{[6-8]}$ Key to the function of NIMS is the Incident Command System (ICS), a hierarchal, flexible, scalable system that provides structure and a clear chain-of-command that consolidates disaster goals and how to carry those out. The Hospital Incident Command System (HICS) was developed to bring ICS structure and coordination to hospital settings and is often composed of hospital administration and nursing leadership but does not have any specified role for physicians. ${ }^{[9]}$

\subsection{Physician Involvement in the Hospital Incident Com- mand System (HICS)}

There are several roles in the HICS that should be filled by physicians. In the context of a pandemic an Infectious Disease expert should fill the Med/Tech position as part of the Command Staff. The direct supervision of the medical provider staff should be an operational position consolidated by the Medical Staff Branch Director. This position is often filled by the Chief Medical Officer for the institution (or designee). The PCSS is a support position to the Medical Staff Branch Director.

\subsection{Description of the Novel Physician Clinical Support Supervisor (PCSS)}

We believe that hospital medicine is uniquely positioned to have a vital role in emergency preparedness, response, and recovery. Hospitalists have an inherent systems-level view as part of their direct and indirect patient care. ${ }^{[10]}$ Building on our prior work, we implemented a novel position, the PCSS, under the Medical Staff Branch Director under the Operations Chief in the HICS organizational chart (see Figure 1), starting as part of the planning phase to our response to COVID-19 in the month leading up to the first case clusters in the United States. While this role was initially filled by a lead hospitalist for emergency preparedness once operational physicians in other specialties were able to carry out the PCSS role throughout the disaster. All physicians who served in the PCSS role held hospital leadership positions (e.g. Assistant Director of Emergency Preparedness, Physician Advisor, elected Medical Staff leadership, Chief Quality Officer, and Chief Medical Informatics Officer) and thus had institutional knowledge and stature that facilitated their effectiveness in this role. Understanding of systems and quality improvement were more important skill sets than in-depth knowledge about COVID-19.

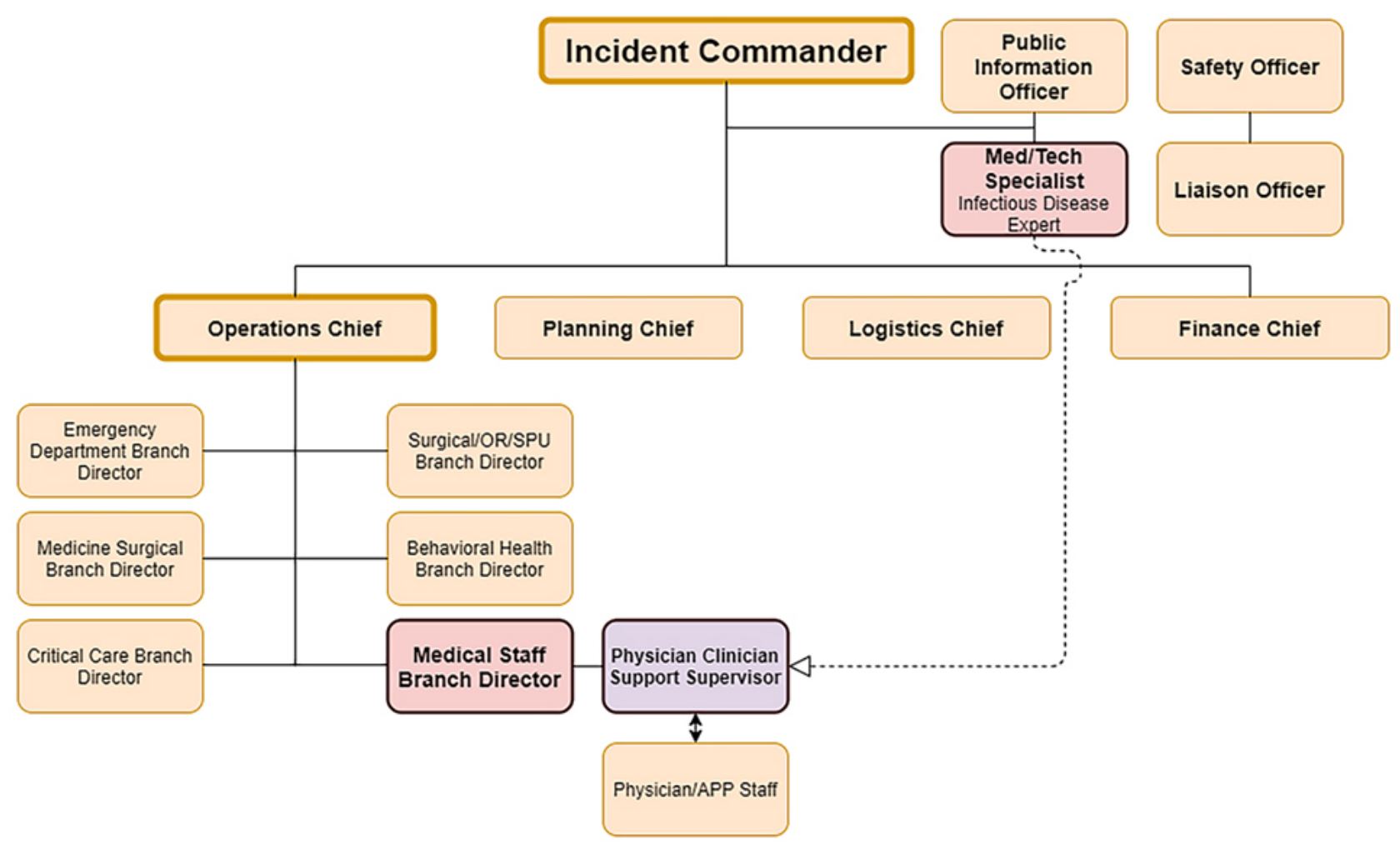

Figure 1. Revised HICS with the novel PCSS position highlighted in relation to the Medical Staff Branch Director with input from the Med/Tech Infectious Disease Expert 


\subsection{Implementation of the PCSS role}

The initial phase of preparation was dedicated to work functioning as a key liaison to clinical groups about the response plans being developed at the upper levels of the hospital administration (Incident Commander, Command Staff, and other Branch Directors). For example, one of the incident command goals was to begin thinking about how units would respond to employee illness affecting tiers from 5\%-25\% of staff. While nursing administration proved very adept at this, physician groups-hospitalists and those with inpatient primary services such as Family Medicine, Cardiology, and the ICU—had less experience with this planning and required PCSS guidance in positing about how to accommodate service staffing needs over time. Dissemination of recommendations from subject matter experts early in the pandemic allowed the PCSS to centralize and consolidate experiences from other institutions and to help quell the rush of misinformation and rumor by funneling information through the PCSS.

\section{Discussion}

\subsection{PCSS role as liaison}

The PCSS continues to remain the main coordinator of large system changes affecting multiple services and lines. Physicians filling this role are available for 24 hour shifts with the number of in-person hours varying based on number and acuity of ongoing needs. The PCSS has a broad footprint in operations. These functions vary in intensity, complexity, and priority, and vary day-to-day. Some examples of critical functions include fielding frontline provider (physician, advanced practice provider, and nursing) phone calls with questions on topics ranging from testing protocols to changes in PPE recommendations; 2) determining for which patients it is appropriate either to apply or remove isolation precautions; and 3) coordinating with the bed board to ensure cohorting of appropriate patients in the correct areas of the hospital.

\subsection{PCSS role as coordinator}

In addition, the PCSS meets daily with leadership of the critical care and hospital medicine COVID-19 teams and units to evaluate barriers to providing high-quality, efficient patient care. In keeping with the need for social distancing while maintaining a reliable platform for building out a large repertoire of source material, we utilized Microsoft Teams ${ }^{\mathrm{TM}}$ for ensuring loop closure and steps for follow up, and for scheduled video conferencing. Concerns and suggestions are fed back to the hospital command center and the incident commander to identify solutions in real time. In turn, solutions, protocols, and added resources are fed back to the frontline teams caring for patients daily for loop closure. By utilizing

Published by Sciedu Press the Teams ${ }^{\mathrm{TM}}$ platform the Medical Staff Branch Director was able to track issues, act when needed, and facilitate the role of the PCSS in resolving issues.

\subsection{PCSS role as advocate}

Frequently during a sustained incident response, clinicians feel a sense of disconnect from the perceived invisible hand of the incident command administration. Here the PCSS acted as an advocate - a central knowledgeable clinician with a defined role in the command center who was available 24/7 to help consolidate and interpret physician, APP, and nursing concerns and funnel them into the command structure as an active member of the Operations Branch under the Medical Staff Branch Director.

The PCSS and Medical Staff Branch Director roles are highly synergistic and complementary. By serving as the point of contact for the front-line teams caring for COVID-19 patients, the PCSS facilitated the ability of the Medical Staff Branch Director to provide higher level strategic and operational oversight for hospital incident command activities. This advocate role also served to prioritize consistent themes (most commonly in response to changes in personal protection equipment (PPE) and viral testing) so that subject matter experts in Infectious Disease could develop messaging and support for emerging science that often brought staff discomfort. Clinicians valued a physician to whom they could report their concerns who was able to share those concerns with the command center structure and provide loop closure as concerns were addressed. Regular interaction with relevant service line team leaders provided a critical structure for transparent communication with those on the front lines. As a single point of contact for two-way communication, questions normally sent broadly to different specialists or teams could instead be routed exclusively to the PCSS to solve up and down the chain-of-command.

\section{Conclusion}

The PCSS role has been an invaluable component of the HICS structure in the position's capacity to act as the liaison, advocate, and coordinator between a highly functioning command system and those who must carry out the mission with confidence. The discoveries garnered from the daily communication between the command and the front-line providers caring for COVID-19 patients facilitated real-time interventions up and down the chain of command improving communication and clarity in a manner not previously discussed in HICS. As part of an all-hazards approach to emergency preparedness, hospitalists play a role that has proven critical within HICS at our institution and should be considered broadly both during the current pandemic, and in planning for disasters in general. 


\section{CONFLicts OF INTEREST Disclosure}

The authors declare they have no conflicts of interest.

\section{REFERENCES}

[1] Homeland Security Presidential Directive-5. 2003

[2] Born CT, Briggs SM, Ciraulo DL, et al. Disasters and mass casualties: I. General principles of response and management. J Am Acad Orthop Surg. 2007; 15(7): 388-96. PMid: 17602028. https://doi.org/10.5435/00124635-200707000-00004

[3] Born CT, Briggs SM, Ciraulo DL, et al. Disasters and mass casualties: II. explosive, biologic, chemical, and nuclear agents. J Am Acad Orthop Surg. 2007; 15(8): 461-73. PMid: 17664366. https://doi.org/10.5435/00124635-200708000-00003

[4] Christian MD, Hawryluck L, Wax RS, et al. Development of a triage protocol for critical care during an influenza pandemic. CMAJ. 2006; 175(11): 1377-81. PMid: 17116904. https://doi.org/10.150 $3 / \mathrm{cmaj} .060911$

[5] Persoff J, Ornoff D, Little C. The Role of Hospital Medicine in Emergency Preparedness: A Framework for Hospitalist Leadership in Disaster Preparedness, Response, and Recovery. J Hosp Med. 2018; 13(10): 713-718. PMid: 30261086. https://doi .org/10.12788 /jhm.3073
[6] Dichter JR, Kanter RK, Dries D, et al. System-level planning, coordination, and communication: care of the critically ill and injured during pandemics and disasters: CHEST consensus statement. Chest. 2014; 146(4 Suppl): e87S-e102S. PMid: 25144140. https://doi.org/10.1378/chest.14-0738

[7] Thomas TL, Hsu SB, Kim HK, et al. The incident command system in disasters: evaluation methods for a hospital-based exercise. Prehosp Disaster Med. 2005; 20(1): 14-23. PMid: 15748010. https://doi.org/10.1017/S1049023X00002090

[8] Agency, F.E.M. NIMS and the Incident Command System. Nov 23, 2004; Available from: https://www.fema.gov/txt/nims/nims _ics_position_paper.txt

[9] Backer H. Hospital Incident Command System Guidebook, 5th ed. Smiley and L. Schoenthal, Editors. California Emergency Medical Services Authority. May 2014. Available from: https://emsa.ca.gov/wp-content/uploads/sites/47/20 17/09/HICS_Guidebook_2014_11.pdf

[10] Inpatient specialists help cut costs, reduce LOS. Hospitalists partner with case managers. Hosp Case Manag. 1997; 5(5): 79-81. 